\title{
0 projeto enquanto jogo: colaboração digital livre?
}

Design as a game: free digital collaboration?

\author{
> Gilfranco Alves \\ Universidade Federal de Mato Grosso do Sul \\ gilfranco.alves@ufms.br
}

\author{
> Anja Pratschke \\ Universidade de São Paulo \\ pratschke@sc.usp.br
}

\begin{abstract}
The paper will present one specific aspect of the PhD research called Cibersemiótica e Processos de Projeto: Metodologia em Revisáo, funded by FAPESP, which in turn is linked to the Nomads.usp research group of the University of São Paulo. The paper discusses aspects of digital design processes within an approach on social collaboration with digital mediation, from concepts based on Actor Network Theory, developed by the French Philosopher Bruno Latour, Swarm Behaviour, which is observed in nature, as well as the Multi-Agent Systems. The paper aims to examine collaborative procedures and hybrid design methods involving several actors or players, trying to redefine the possibilities of conception in Architecture with a bottom-up approach, considering the concept of game applied to digital design processes.
\end{abstract}

Keywords: Actors Network Theory; Cybersmiotics; games;Multi-Agent System; Swarm Behaviour.

\section{Introdução}

O uso de computadores e de jogos computacionais tem promovido significativamente o uso de valores numéricos como representação de ideias. Ao se apertar um botáo, por assim dizer, as escolhas de projeto são convertidas para uma quantidade de diferentes parâmetros, o que permite que o potencial para melhorias que possam ser implementadas em outros contextos torne-se mais visível e eficiente dentro do processo de projeto.

Bart Lootsma ${ }^{1}$ afirma que os meios digitais e especialmente os recentes desenvolvimentos no campo dos jogos computacionais estão influenciando significativamente o modo como ampliamos as possibilidades de experenciar as cidades (LOOTSMA, 2007). Seria entấo, a colaboração em rede, somadas à potencialidade dos jogos eletrônicos on line e em tempo real, uma possibilidade significativa para os processos digitais de projeto?

Partimos da premissa de que processos digitais de projeto podem adquirir qualidades interativas, e portanto, expressar cada vez mais demandas coletivas. Ainda que talvez nunca se atinja uma estratégia cem por cento do tipo bottom-up (de baixo para cima), em função de nossa capacidade intuitiva, e também porque em algum momento é necessário existir um responsável pela tomada de decisão, acreditamos que à medida que os processos se tornem

1 Bart Lootsma (nascido em 1957 em Amsterdá, Holanda) estudou arquitetura na Universidade de Tecnologia de Eindhoven, durante 1975-1984. Ele é um historiador, crítico e curador nas áreas de arquitetura, design e artes plásticas. Fonte: http://en.wikipedia.org/wiki/Bart_Lootsma. Acessado em 31/07/2014. menos do tipo top-down (de cima para baixo), estaremos dando um grande passo em direção à processos mais interativos e mais comprometidos com valores sócio-culturais coletivos.

Este artigo pretende comparar brevemente alguns aspectos dos processos digitais de projeto dentro de um recorte social de colaboração com mediaçáo digital, a partir de conceitos advindos de três teorias, a Teoria de Atores em Rede (Actor-network Theory) desenvolvida pelo filósofo francês Bruno Latour ${ }^{2}$, a Teoria do Comportamento de Enxame (Swarm Behaviour ${ }^{3}$ ) observado na natureza e pesquisado inicialmente pelo especialista em vida artificial e computação gráfica, Craig Reynolds, e a Teoria de Sistemas de Agentes Múltiplos (Multi-agent Systems).

\section{Colaboração e mediação digital a partir de um recorte transdisciplinar}

Tradicionalmente os processos de projeto se utilizam da intuição e da inspiração, e decisóes do tipo top-down muitas vezes são realizadas

2 Bruno Latour (francês: nascido 22 de junho de 1947) é um sociólogo da ciência e antropólogo francês. Ele é especialmente conhecido por seu trabalho no campo da ciência e estudos da tecnologia (STS). Fonte: http://en.wikipedia.org/wiki/Bruno_Latour, Acessado em 31/07/2014.

3 Comportamento Swarm, ou swarming, é um comportamento coletivo exibido por animais de tamanho similar que se agregam juntos, talvez agindo sobre o mesmo local, ou talvez em movimentos em massa ou migrando em alguma direção. Como termo, enxame é aplicada especialmente para os insetos, mas também pode ser aplicado a qualquer outro animal que apresenta um comportamento de bando. Fonte: http:// en.wikipedia.org/wiki/Swarm_behaviour. Acessado em 31/07/2014. 
com informaçóes incompletas, imprecisas e mesmo, impositivas. Desejamos que os desdobramentos possibilitados com a discussão proposta, apontem alternativas que melhorem, não apenas a performance como resultante dos projetos, mas também a eficiência na obtenção de dados que servirão de parâmetros para os projetos e, antes ainda, para predefiniçóes em nível do que é conhecido como metadesign, (VASSÃO, 2010) a serem aplicadas em processos digitais de projetos.

É de suma importância para os processos de projeto, justamente definir o design do design previamente com os atuadores do processo. Acreditamos que é a partir da participação de todos os envolvidos no desenho das açóes que o processo de projeto pode se tornar verdadeiramente colaborativo e que conceitos como a interatividade e a imprevisibilidade podem ser previstos e parametrizados.

A Teoria de Atores em Rede (Actor-Network Theory ou ANT), também conhecida como teoria da inscrição ou a sociologia da tradução, surgiu durante meados dos anos 1980, principalmente com o trabalho de Bruno Latour, Michel Callon ${ }^{4}$ e John Law ${ }^{5}$. De acordo com Latour (2005), a ANT propóe uma atuaçáo livre, compatível com o conceito de auto-organização, na qual se torna mais fácil encontrar ordem depois de se ter permitido os atores ou atuadores implantarem toda uma gama de controvérsias na qual estáo imersos. A ideia da controvérsia é central na proposta da ANT. Para produzir e recuperar algum sentido de ordem, Latour propóe que a melhor solução é traçar conexôes entre as próprias controvérsias ao invés de tentar decidir como resolver qualquer controvérsia. A busca por ordem, rigor e padrão não é abandonada. É simplesmente traduzida um passo a mais em direção à abstração, de modo que os atuadores estấo autorizados a revelar os seus próprios universos, não importa o quão intuitivo se mostrem.

De acordo com a definição apresentada na Wikipedia ${ }^{6}$, o comportamento em enxame, Swarm, ou Swarming, é um comportamento coletivo exibido por animais de tamanho similar que se agregam e atuam em conjunto, às vezes agindo sobre o mesmo local, em movimento, às vezes em massa ou ainda, migrando em alguma direçáo. De um modo geral, podemos dizer que de modo complementar à atuação em rede definida por Latour, este comportamento apresenta características evolutivas, de emergência e de auto-organização.

Os primeiros estudos do comportamento em enxame empregaram modelos matemáticos para simular e entender o seu comportamento. Os modelos mais simples de instruçóes algorítmicas representam geralmente animais específicos, seguindo, por exemplo, três regras:

1. Movimente-se na mesma direção que seus vizinhos

$4 \quad$ Michel Callon é professor de Sociologia na "École des Mines de Paris" e investigador no "Center for the Sociology of Innovation". Fonte: http:// pt.wikipedia.org/wiki/Michel_Callon. Acessado em 31/07/2014.

5 John Law é um sociólogo atualmente na Faculdade de Ciências Sociais da Universidade Aberta e proponente-chave da teoria de atores em rede. Fonte: http://en.wikipedia.org/wiki/John_Law_(sociologist). Acessado em 07/09/2014.

6 http://en.wikipedia.org/wiki/Swarm_behaviour. Acessado em 31/07/2014.
2. Fique perto de seus vizinhos

3. Evite colisóes com seus vizinhos

O programa de computador Boids, criado por Craig Reynolds ${ }^{7}$ em 1986, foi o primeiro a simular o comportamento de enxame seguindo as regras acima.

Levar em consideração um comportamento de enxame para questóes arquitetônicas de adaptaçáo espacial significa, não só atender às necessidades individuais de seus usuários, mas também resolver os interesses e conflitos existentes entre esses múltiplos usuários. Estes conflitos poderiam ser resolvidos de um ponto de vista ideal encontrando soluçóes espaciais que satisfaçam a todos. Em muitas situaçóes, no entanto, este objetivo pode não ser possível. Nesse caso, certas atividades ou determinados usuários precisariam ser priorizados em detrimento de outros, criando certos pontos de atração, ou focos de interesse.

$\mathrm{O}$ arquiteto holandês Kas Oosterhuis ${ }^{8}$ vem atuando há vários anos no desenvolvimento de arquiteturas não padronizadas (non-standard architectures) com estratégias que envolvem concepçóes de projeto em redes de interação e responsividade. Oosterhuis afirma que em um processo colaborativo em rede os agentes atuam como pássaros em bando (Figura 1), onde cada agente é um nó em uma rede interconectada desempenhando uma regra específica, produzindo assim uma rede de fluxo de informaçóes multidirecional, ou seja, um sistema de agentes múltiplos.

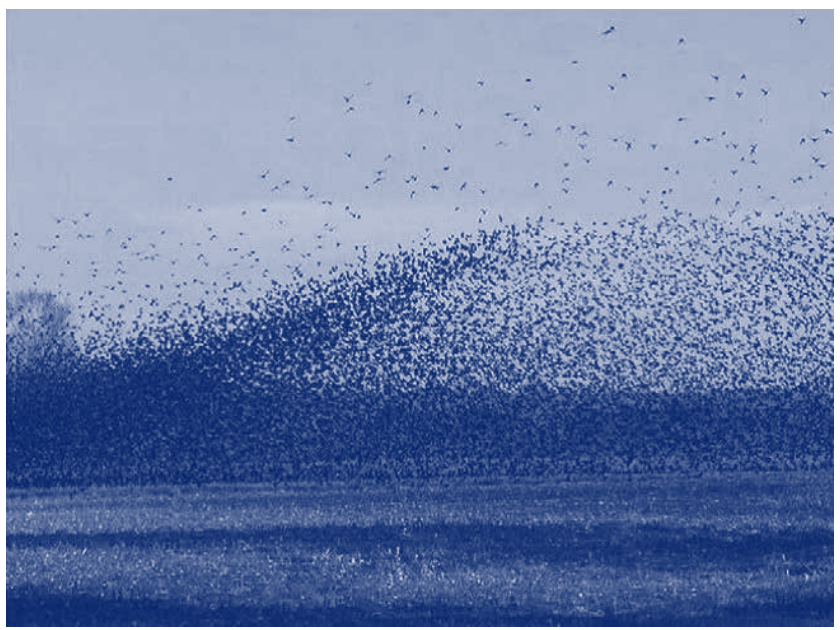

Figura l: comportamento em enxame (swarm behaviour). Fonte: Oosterhuis, 2012.

7 Craig W. Reynolds (nascido em 15 de março de 1953), é especialista em vida artificial, e de aplicaçóes gráficas que criou a simulação de vida artificial Boids em 1986. Fonte: http://en.wikipedia.org/wiki/ Craig_Reynolds_(computer_graphics). Acessado em 07/08/2014.

8 Kas Oosterhuis é professor da Faculdade de Arquitetura da Universidade Técnica de Delft, bem como diretor do Hyperbody e do Protospace Laboratory for Collaborative Design and Engineering. Seu ensino e pesquisa são nas áreas de arquitetura interativa, comportamento em tempo real de edifícios e ambientes, conceitos de edifícios vivos, design colaborativo, produção file-to-factory e design paramétrico. Fonte: http://en.wikipedia.org/wiki/Kas_Oosterhuis. Acesso em 06/08/2014. 
Este processo dirigido à informação ou à troca de informaçóes a partir de referências, pode ser fundamentado na Teoria de Atores em Rede de Bruno Latour com vistas à construção de um método para processos digitais de projeto. Segundo Oosterhuis, este modo de se definir o processo digital de projeto baseado na Teoria de Atores em Rede, requer uma mentalidade diferente, onde deve-se entender que tudo, em princípio, é dinâmico - e não, estático.

Você precisa sentir esta evolução interna. Porque tudo evolui. Seu projeto evolui, situaçôes evoluem, tudo evolui. Então você funciona evoluindo internamente. De novo, como um ponto no Swarm que pode agir somente dentro do Swarm, ele náo pode ser tomado sozinho. Como você com seu cérebro, você não pode agir sozinho, porque seu cérebro é inútil se você não está conectado com outros cérebros. Talvez você nem mesmo pense. Você não veja nada. Porque tudo que evolui é baseado em conectividade, tudo é baseado em conectividade, no final. Eo bi direcionamento, em questão, é somente entre dois pontos, mas claro, é multidirecional, porque há muitos jogadores, muitos tipos de componentes interativos, Swarms interativos, grupos interativos. Mas esta é a complexidade que surge quando você começa do básico, então você tem que mergulhar internamente, começar deste ponto, definir suas regras e então, trabalhar a partir dai. (OOSTERHUIS, apud ALVES 2013).

Estabelece-se um processo de projeto onde o próprio projeto pode ser representado por números e ao mesmo tempo, ser influenciado por eles. Em um processo orientado à performance, a realidade é fornecida e não meramente observada. Para Yang (2008), "sistemas de agentes múltiplos são sistemas compostos de vários agentes autônomos. Estes agentes podem utilizar um modelo de crença-desejo-intenção ou outros mecanismos para orientar comportamentos, responder ao ambiente, ou se comunicar e interagir com outros agentes." (YANG, 2008 p. 10. Tradução nossa9).

O elemento principal de um sistema de agentes múltiplos é um "agente inteligente". Agentes inteligentes são entidades autônomas que observam e agem sobre seu ambiente e direcionam suas atividades para alcançar determinados objetivos. Assim, podemos considerar que Sistemas Multi-Agentes (MAS) consistem de uma série de agentes autônomos que podem se comunicar uns com os outros, permitindo um elevado grau de flexibilidade e reconfigurabilidade. Além de estarem fortemente relacionados ao conceito de Swarm e, portanto, à Teoria de Atores em Rede de Latour, também seria possível, dentro destes vários níveis múltiplos de agenciamento, propor uma abordagem Cibernética, onde sistemas de feedback loops também poderiam ser incorporados. Isto significa que eles poderiam permanecer num processo constante de adaptação, mais condizentes com processos evolutivos e com a própria dinâmica da vida. Isso dotaria os agentes de uma inteligência que os capacitaria a saírem

9 Do original em Inglês: "Multi-Agent Systems are systems composed of several autonomous agents. These agents may use belief-desire-intention model or other mechanisms to guide behaviours, respond to the environment, or communicate and interact with other agents." de uma condição apenas reflexiva em relação ao seu ambiente para evoluírem para uma condição de aprendizado e evolução.

Para Oosterhuis e Jaskiewicz ${ }^{10}$ (2007), projetar em arquitetura é um jogo no qual o objetivo é criar um edifício. É um jogo no qual os arquitetos precisam jogar de acordo com as regras da física, da economia e da sociedade, por exemplo. Ainda segundo os autores, projetar é por natureza um jogo com múltiplos jogadores, no qual muitos especialistas necessitam trabalhar em conjunto para aumentar suas perspectivas de vencer.

Para os autores, mais importante do que todas as questóes técnicas sobre como fazer as coisas funcionarem é definir qual o modo de se projetar no qual estas estratégias implicam: "Computadores nos permitem jogar juntos em tempo real. Em um jogo multiplayer, a troca de informaçôes acontece instantaneamente, muitos ciclos por segundo. O que vai acontecer se uma informação de projeto é trocada imediatamente entre designers diferentes?" (OOSTERHUIS E JASKIEWITZ, 2007. p. 359. Traduçáo nossa ${ }^{11}$ ). Especulamos que provavelmente haverá modificaçôes na metodologia que envolve o processo de projeto. Provavelmente também, simultaneamente às contribuiçóes intuitivas, do tipo de cima para baixo (top-down), em cada subsistema, haverá muitos níveis de colaboraçáo do tipo de baixo para cima (bottom-up) no sistema como um todo.

Deste modo, parece-nos relevante perguntar: o que temos a ganhar com o uso de jogos para propostas arquitetônicas? De acordo com Hovestadt ${ }^{12}$ (2007), o discurso arquitetônico ainda vigente no começo do século XXI é baseado em concepçóes obsoletas das realidades tecnológicas. $\mathrm{O}$ autor aponta que muitas tentativas realizadas por arquitetos e designers de jogos, no sentido de produzir hipóteses e teorizar sobre jogos arquitetônicos foram muitas vezes superficialmente exploradas e apresentadas e, assim, isto tornou-se um argumento para os arquitetos mais conservadores rotularem esta vertente como um "gênero amador". No entanto, é sabido que por meio dos jogos, foram desenvolvidos métodos e tecnologias eficazes para sistemas mais complexos e dinâmicos de modelagem, controle e interação. Estes sistemas, muitas vezes, podem alcançar e simular muito mais do que seria possível na prática arquitetônica cotidiana.

Os jogos digitais aplicados em processos de projeto, podem explorar estes aspectos e propor ambientes que simulem vários comportamentos, estimulando estratégias colaborativas onde os atuadores podem influenciar e serem influenciados por todos os participantes do jogo. Estas possibilidades podem conferir ao

10 Tomasz Jaskiewicz é um arquiteto, urbanista, pesquisador acadêmico e educador. Depois de terminar seus estudos de graduação em Arquitetura e Urbanismo na TU Gdansk, Polônia, ele se juntou ao grupo Hyperbody na TU Delft, Holanda, onde obteve em 2005, o grau de Master of Science in Architecture. Fonte: http://www.hyperbody.nl/about/who/. Acesso em 16/08/2014.

11 Do original em Inglês: "Computers let us play together in real time. In a multiplayer game, the Exchange of information happens instantly, many cycles per second. What will happen if desing information gets exchanged immediately between different designers?"

12 Ludger Hovestadt é Professor de Projeto de Arquitetura Assistido por Computador (CAAD) do Swiss Federal Institute of Technology (Eidgenössische Technische Hochschule, ETH) em Zurique. Fonte: http://www. caad.arch.ethz.ch/blog/ludger-hovestadt/. Acesso em 16/08/2014. 
processo de projeto características mais interativas e tornar mais claros os objetivos a serem atingidos.

Jogos como o SimCity (1989), The Sims (2000), ambos da Maxis, e o Minecraft, criado por Markus Persson para a plataforma Xbox, e que atualmente vem sendo desenvolvido pela University of Southern California's School of Architecture em uma versão (Figura 2) que promete potencializar as possibilidade de se planejar uma cidade, oferecem uma maior participação colaborativa entre os usuários e são exemplos de como os jogos podem simular situaçóes mais dinâmicas e interativas dentro de processos digitais de projeto.
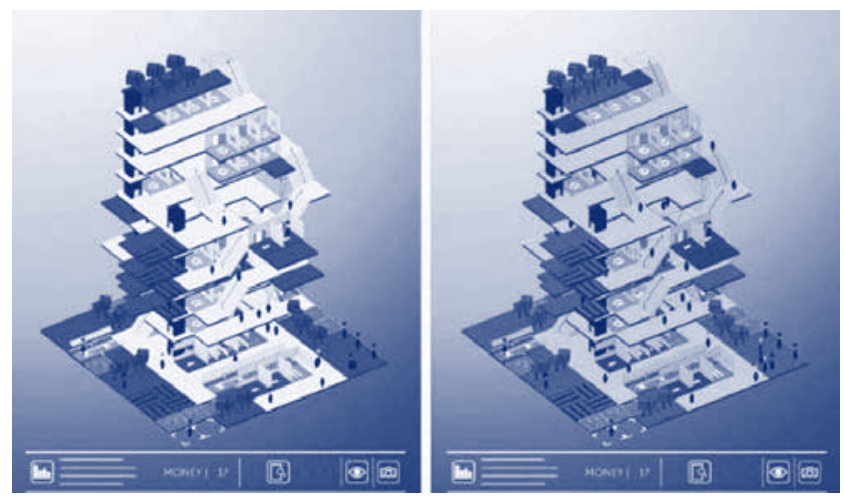

Figura 2: Minecraft for real life. Fonte: http://www.fastcoexist.com/3034872/ minecraft-for-real-life-this-video-game-wants-to-help-redesign-actual-cities\#3

Nosso interesse está na busca do desenvolvimento e do estudo de sistemas que possibilitem benefício mútuo através do qual estruturas arquitetônicas fundamentais possam estar ligadas à modelagem de jogos, produção e padróes de interação entre usuários. Essas interfaces poderiam estar voltadas para o uso diário dos escritórios de arquitetura, porém sabemos que aprofundar os níveis de interação demanda uma quantidade significativa de trabalho, treinamento e paciência para que o processo possa ser compreendido e como consequência, evoluir.

\section{Considerações finais}

Acreditamos que as teorias relacionadas possuem aspectos que são complementares e que podem constituir-se em uma fundamentação importante para processos digitais de projeto. A noção de atuadores proposta por Latour é a base para a concepção de sistemas de agentes múltiplos, que por sua vez, também se utilizam de estratégias inspiradas a partir do comportamento de enxame.

Em geral os jogos operam dentro de um mesmo princípio: eles são manifestaçôes técnicas que descrevem meios de se pensar, refletem escolhas e, via de regra, são mais poderosos e adaptáveis que as técnicas de construção disponíveis, uma vez que estas após atingirem um certo limite de qualidade, muitas vezes permanecem no mesmo status sem atualização ou evolução de performance. (HOVESTADT, 2007). Esta situação poderia levar a certa acomodação em relação aos usuários, justamente por não propor desafios. Já em uma situação simulada, os limites podem ser expandidos pela condição interativa dos modelos híbridos. Os ambientes não sáo operados apenas externamente, como em uma rede de simples causa e efeito, mas sim de modo interativo, desde que o usuário esteja incorporado ao sistema, afetando e sendo afetado por outros. Desse modo, a experiência híbrida entre o ambiente digital e o físico torna-se táo intensa que acaba por dissolver em muitos sentidos os limites da experiência do jogo.

\section{Agradecimentos}

Ao Comitê Internacional SIGRADI por fornecer a base para este modelo.

Á FAPESP - Fundação de Amparo à Pesquisa do Estado de São Paulo. (http://www.fapesp.br/)

Ao grupo de pesquisa Nomads.usp. (www.nomads.usp.br).

\section{Referências}

ALVES, Gilfranco (2013). Entrevista com Kas Oosterhuis: Conversa sobre o Hyperbody, arquitetura interativa, design paramétrico e processos de projeto e de produção contemporâneos. Entrevista, São Paulo, ano 14, n. 054.01, Vitruvius, maio 2013. < http://www.vitruvius.com.br/revistas/ $\mathrm{read} /$ entrevista/14.054/4758 >.

HOVESTAD, Ludger (2007). Why Games for Architecture? pp. 335-339. In BORRIES, Friedrich von; WALZ, Steffen P.; BÖTTGER, Matthias. (Ed.). Space Time Play: Computer Games, Architecture and Urbanism: The Next Level. Basel: Birkhäuser.

LATOUR, Bruno (2005). Reassembling the Social: An Introduction to ActorNetwork Theory. New York: Oxford University Press.

LOOTSMA, Bart (2007). Toward a Game Theory of Architecture. pp. 404-406. In: BORRIES, Friedrich von; WALZ, Steffen P.; BÖTTGER, Matthias. (Ed.). Space Time Play: Computer Games, Architecture and Urbanism: The Next Level. Basel: Birkhäuser.

OOSTERHUIS, Kas; JASKIEWICZ, Thomasz (2007). 798 Multipayer Design Game. pp. 358-361. In BORRIES, Friedrich von; WALZ, Steffen P.; BÖTTGER, Matthias. (Ed.). Space Time Play: Computer Games, Architecture and Urbanism: The Next Level. Basel: Birkhäuser.

VASSĀO, Caio Adorno (2010). Metadesign: ferramentas, estratégias e ética para a complexidade. São Paulo: Blucher.

YANG, Ang (2008). Intelligent Complex Adaptive Systems. London: IGI Publishing. 